\title{
Abschätzung des Risikos von Naturgefahren für Wasser- versorgungssysteme auf Basis der Systemvulnerabilität
}

\author{
Assessing the risk of natural hazards to water-supply systems on the basis of \\ system vulnerability
}

von M. Möderl, S. De Toffol, D. Vanham, E. Fleischhacker und W. Rauch

\section{Kurzfassung/Summary}

Die Gefährdung von Wasserversorgungssystemen in Bezug auf Naturgefahren wird bislang - wenn überhaupt - nur durch eine räumlicheVerschneidungder Gefahrenzonen mit dem Leitungsnetzwerk bestimmt. Im Folgenden wird eine innovative Methodik präsentiert, bei welcher die Systemvulnerabilität der Wasserversorgung quantifiziert wird. Dabei wird für jede Komponenten bestimmt, welche Auswirkungen der Ausfall dieser Komponente auf das Gesamtsystem aufweist. Die Berechnung des Systemvulnerabilität erfolgt dabei mit dem am Arbeitsbereich Umwelttechnik, Universität Innsbruck entwickelten Softwarewerkzeug VulNetWS (Vulnerability of Water Supply Networks). Die Anwendung der Methodik an einer überregionalen alpinen Fallstudie ergab, dass im Untersuchungsgebiet 0,42 von $21,375 \mathrm{~km}^{2}$ ein $\mathrm{Ri}$ sikopotential aufweisen und für weiter detaillierte Studien empfohlen werden.

The risks to water-supply systems from natural hazards has been determined up to now, if at all, only from the overlaps between risk zones and the distribution system. This article presents an innovative method of quantifying the vulnerability of water supply systems. This provides, for each component, determination of the impact of its potential failure on the overall system. System vulnerability is calculated by use of the software tool VulNetWS (Vulnerability of Water Supply Networks) developed by Unit of Environmental Enginee- ring domain of University Innsbruck. Application of this method in a supraregional Alpine case stu$d y$ has shown that $0.42 \mathrm{~km}^{2}$ out of the $21.375 \mathrm{~km}^{2}$ study area exhibits some risk potential and this justifies detailed study.

\section{Einleitung}

Die Ansprüche der Bevölkerung an die Versorgungssicherheit in Bezug auf Trinkwasser sind sehr hoch und bereits kurzfristige Ausfälle von Teilbereichen des Leitungsnetzes werden von den KonsumentInnen kritisch gesehen. Die Quantifizierung der potentiellen Beeinträchtigung der Wasserversorgungssysteme durch äußere Einwirkungen wie z. B. Naturgefahren ist daher ein relevantes Thema für die Betreiber. Üblicherweise wird dazu eine vereinfachte Methodik angewandt, bei welcher die von Naturgefahren betroffenen Flächen (Gefahrenzonen) mit dem Wasserversorgungsnetzwerk räumlich verschnitten werden. Dadurch werden zwar jene Teilbereiche des Systems identifiziert, die potentiell beeinträchtigt werden - die Auswirkungen auf das Gesamtsystem können damit aber nicht bestimmt werden.

In dieser Arbeit wird eine innovative Methodik zu diesem Thema vorgestellt. Dabei wird in einem ersten Schritt die Systemvulnerabilität des Wasserversorgungsnetzes bestimmt. Nach Ezell, 2007 bezeichnet die Vulnerabilitätsbeurteilung die Identifikation von Schwachstellen im System unter Berücksichtigung von bestimmten Gefahren die die Aufgabe bestimmter Systemkomponenten beeinträchtigen. Gemäß dieser Definition wird auf der Basis von hydraulischen und quantitativen Simulation des Systems der Ausfall jeder einzelnen Komponente simuliert und mittels einer Bewertungsfunktion quantifiziert. Damit kann für jede Leitung, Pumpe, Quelle, etc. angegeben werden, welche Auswirkungen auf das Gesamtsystem entstehen, wenn eben diese Komponente ausfällt. Aus diesen Informationen kann nun wieder eine räumliche Systemvulnerabilität bestimmt werden, bei welcher jeder Teilbereich des Systems gemäß der vorgängig berechneten Vulnerabilität eingestuft wird. Für die Bestimmung der Systemvulnerabilität wurde am Arbeitsbereich Umwelttechnik der Universität Innsbruck das Softwarewerkzeug VulNetWS entwickelt, welches auf Basis von Simulationen mit der bekannten Software Epanet2 (Rossman, 2000) die automatisierte Erstellung von Vulnerabilitätskarten ermöglicht.

Im zweiten Schritt der Methodik werden nun diese räumlichen Informationen mit den Gefahrenzonen verschnitten. Nach Kelman, 2003 kann Risiko als Vulnerabilität mal Gefährdung definiert werden. Vergleicht man diese Definition mit der häufig verwendeten Definition - Risiko ist Schaden mal Wahrscheinlichkeit - kann Schaden mit Vulnerabilität und Wahrscheinlichkeit mit Gefährdung gleichgesetzt werden. Die Definition nach Kelman ermöglicht aber die Kombination von Vulnerabilitäts- und Gefahrenkarte zur Risikokarte. Diese Karte gibt den BetreiberInnen von Wasserversorgungsunternehmen die Möglich- 


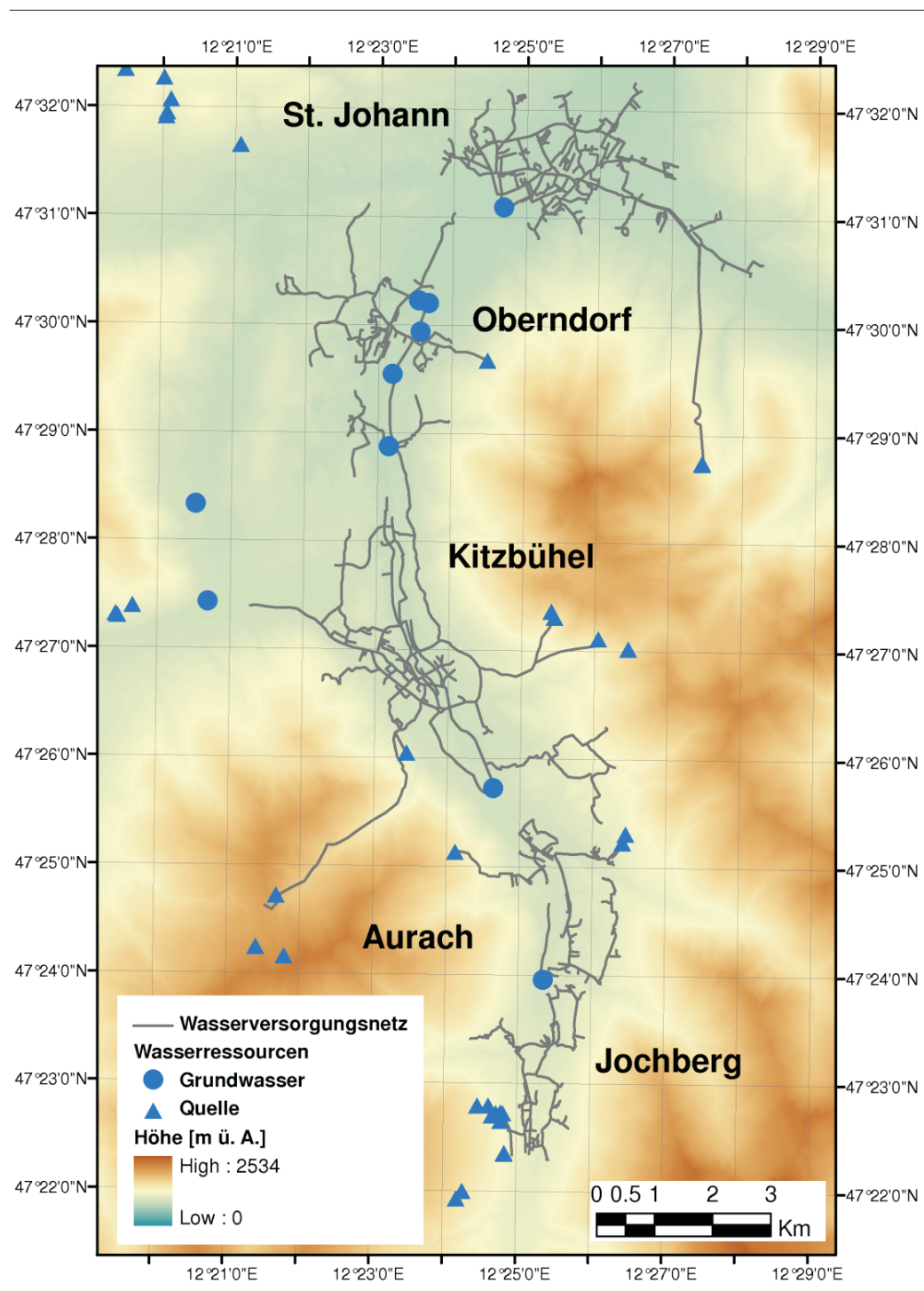

Abb. 1: Karte des Einzugsgebietes mit Geländehöhenrasterdate

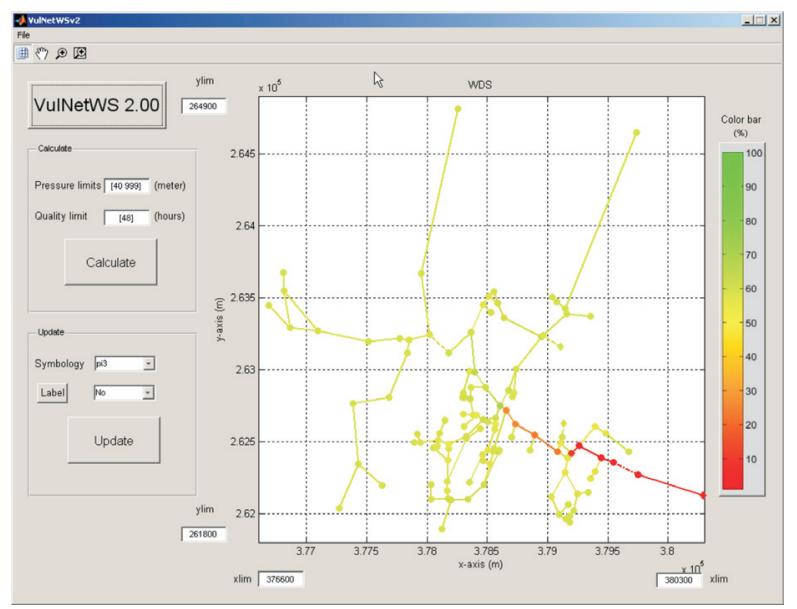

Abb. 2: Grafische Oberfläche der Software VuINetWS keit Gefährdungen quantifizieren zu können und gezielt Präventivmaßnahmen zu treffen.

\section{Methoden}

\subsection{Beschreibung des Unter- suchungsgebietes und der Fallstudie}

Die Fallstudie, anhand derer die Methodik getestet wird, umfasst fünf Gemeinden im Raum Kitzbühel, von denen zwei eher urban und die anderen eher ländlichen Charakter aufweisen. Tourismus ist in dieser Region vorrangig, was sich in 1,6 Mil. Übernachtungen in der Saison 2001 widerspiegelt. Im Gegensatz sind nur etwa 21.200 EinwohnerInnen in der Region beheimatet, was eine starke saisonale Schwankung des Bedarfs hervorruft. Der Trinkwasserbedarf der Gemeinden wird durch Quellfassungen und Grundwasserbrunnen gedeckt und die Versorgungssysteme der fünf Gemeinden sind im Wesentlichen autark. Eine detaillierte Beschreibung der Fallstudie bietet Vanham et al., 2008.

Essentielle Eingangsgröße für eine hydraulische Simulation ist der Wasserbedarf der Gemeinden. Dieser wurde basierend auf der in Vanham et al., 2007 vorgestellten Methodik berechnet. Dabei wird ein von der Statistik Austria vertriebener Rasterdatensatz verwendet, der bei einer 125 x $125 \mathrm{~m}$ Auflösung, die EinwohnerInnenund Beschäftigtenzahlen beinhaltet. Um den Wasserbedarf des Fremdenverkehrs zu berücksichtigen wurde oben beschriebener Raster mit den Nächtigungszahlen beaufschlagt.

Um die Vulnerabilität von Wasserversorgungssystem bestimmen zu können, wurden Modellierungsdaten der Fallstudie erhoben und analysiert (beschrieben in Vanham et al., 2007). Die hydraulischen Modelle der Wasserversorgungssysteme wurden 
anhand der Bedarfsraster mit Betriebsdaten der BetreiberInnen der Wasserversorgungssysteme (erhoben durch einen Fragebogen) kalibriert. Ebenso wurde der Wasserzulauf durch Quellen und Brunnen anhand von Betriebsdaten abgeglichen. Eine Kalibrierung der Druckverteilung im System konnte mangels Messdaten nicht durchgeführt werden ist jedoch empfehlenswert.

\subsection{Ermittlung der Systemvul- nerabilität mittels hydrau- lischer Simulationen}

Die Vulnerabilität der Wasserversorgungssysteme wurde mit einem neu entwickeltem Matlab $^{\odot}$-Tool bestimmt. Das Software Werkzeug VulNetWS (Vulnerability of Water Supply Networks) wird mittels grafischer Oberfläche bedient (siehe Abb. 2). Die Software kann auch ohne Matlab $^{\odot}$-Installation verwendet werden, erfordert dann jedoch die Installation einer Bibliothek die für VulNetWS AnwenderInnen kostenlos erhältlich ist.

VulNetWS berechnet das Systemverhalten von Wasserversorgungen auf Basis hydraulischer Simulationen und bewertet das Verhalten in Bezug auf Vulnerabilität mittels mehreren Parametern (in Möderl et al., 2008 detailliert beschrieben). Eine wesentliche Kenngröße des Systemverhaltens ist z. B. der hydraulische Druck: je mehr Knoten außerhalb eines (frei definierbaren) Bereiches von z. B. 4-10 bar liegen desto gefährdeter wird das System eingestuft. In ähnlicher Art und Weise wird auch die Aufenthaltszeit des Wassers in der Leitung beurteilt: je kürzer desto besser.

Intern simuliert und bewertet VulNetWS die hydraulische Qualität und Wasserqualität bei Ausfall von Systemkomponenten. Basierend auf einem Epanet2 (Rossman, 2000) Inputfile wurden Simulationen für den Ausfall jeder

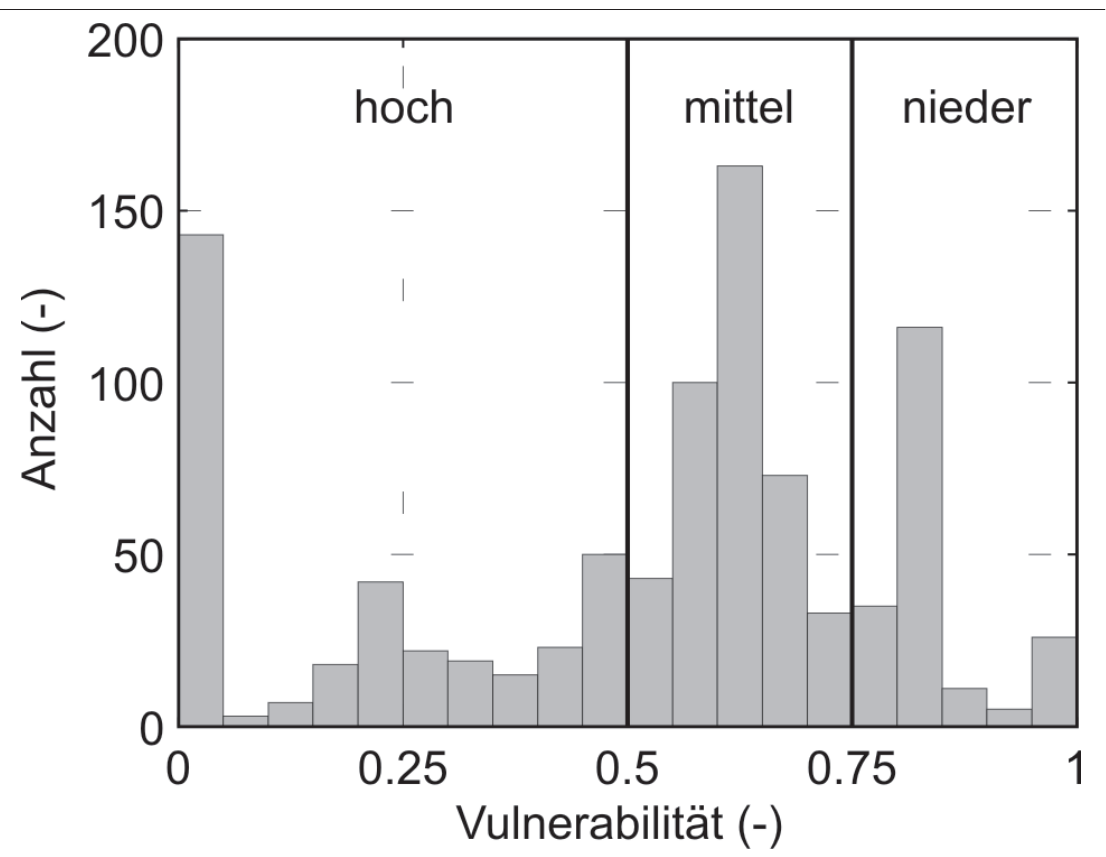

Abb. 3: Reklassifizierung der Vulnerabilität in drei Klassen

einzelner Komponente (hier insgesamt 1.540) berechnet. Jede dieser Simulationen wird hinsichtlich hydraulischer und qualitativer Situation über eine 50-stündige Periode durchgeführt.

Als Resultat erhält man Ergebnisse der Bewertungsfunktion die zwischen 0 (-) und 1 (-) liegen, wobei $1(-)$ als nicht vulnerabel und 0 (-) als stark vulnerabel bezeichnet werden kann. Die Vulnerabilität einer Komponente beziffert in diesem Zusammenhang das Verhalten des Gesamtsystems bei Ausfall eben dieser Komponente: z. B. heißt ein Vulnerabilitätswert für eine Pumpe von 0.23 (-), dass - bei Ausfall dieser Pumpe - nur $23 \%$ des gesamten Wasserbedarfes mit ausreichendem Druck und Wasserqualität bereitgestellt wird.

Im letzen Schritt der Vulnerabilitätsbestimmung wurden die Ergebnisse des Tools VulNetWS in ein geographisches Informationssystem importiert und nach den in Abbildung 3 eingeteilten Klassen Nieder, Mittel und Hoch reklassifiziert. Für die Einteilung der Klassen wurden die Ergebnisse einer früheren Studie herangezogen. In Möderl et al., 2007 wurden 2.280 virtuelle Wasserversorgungsnetze mit unterschiedlicher Netzstruktur generiert. Die mit der Systemvulnerabilitätkorrespondierenden Ergebnisse der Bewertungsfunktion dieser früheren Studie sind als Histogramm in Abbildung 3 hinterlegt.

\subsection{Abschätzung der Natur- gefahren}

Für die qualitative Abschätzung der Gefährdung der Wasserversorgungssysteme werden die in Tabelle 1 aufgelisteten Naturgefahren betrachtet. Hierbei wird angenommen, dass durch Überflutungen die Wasserressourcen verunreinigt werden können bzw. die Elektrik der Pumpen in Tiefbrunnen beeinträchtigt wird. Hangrutschungen und Muren können die Quell- und Brunnenfassungen beschädigen. Hinsichtlich der Bedrohung durch Lawinen wird angenommen, dass durch eine Staub- oder Bodenlawine eine Beschädigung bzw. Kontaminierung von Quellfassungen möglich 
Tab. 1: Evaluierte Naturgefahren und verwendete Datensätze

\begin{tabular}{|c|c|c|c|}
\hline Naturgefahr & Einwirkung & $\begin{array}{l}\text { Fläche abge- } \\
\text { deckt durch Na- } \\
\text { turgefahrenplan }\end{array}$ & andere Flächen \\
\hline h1 - Überflutung & $\begin{array}{l}\text { Wasserressourcen } \\
\text { und Verteilungssystem }\end{array}$ & $\begin{array}{l}\text { Hochwasser- } \\
\text { risikozonierung }\end{array}$ & $\begin{array}{l}\text { keine Evaluierung } \\
\text { durchgeführt }\end{array}$ \\
\hline $\begin{array}{l}\text { h2 - Hangrut- } \\
\text { schung }\end{array}$ & $\begin{array}{l}\text { Wasserressourcen } \\
\text { und Verteilungssystem }\end{array}$ & \multirow{3}{*}{ Gefahrenzonenplan } & \multirow{3}{*}{$\begin{array}{l}\text { digitales Höhen- } \\
\text { modell CORINE } \\
\text { Landnutzung }\end{array}$} \\
\hline h3 - Lawine & Wasserressourcen & & \\
\hline h4 - Mure & $\begin{array}{l}\text { Wasserressourcen } \\
\text { und Verteilungssystem }\end{array}$ & & \\
\hline
\end{tabular}

ist. Weiters wird angenommen, dass Hangrutschungen und $\mathrm{Mu}-$ ren im Gegensatz zu Lawinen alle Wasserversorgungskomponenten inklusive Leitungen mechanisch angreifen können. Ebenso ist es denkbar, dass bei Überflutung aufgrund des erhöhten äußeren Druckes, Oberflächenwasser bei den Verbindungsmuffen des Leitungssystems eindringen kann.

Die Erstellung der Gefahrenkarten erfolgt vornehmlich auf Basis des existierenden Gefahrenzonenplans, der im BGBl 436/1976, 1976 geregelt ist und der Hochwasserrisikozonierung (Blöschl et al., 2006). Die Gefahrenzonenpläne sind jedoch nicht umfassend erhoben. Damit mussten in Gebieten wo kein Gefahrenzonenplan vorlag alternative Methoden benutzt werden. Für die qualitative Abschätzung der Gefährdung wurden in diesen Zonen GIS - Datensätze (digitales Höhenmodell, CORINE Landnutzung) in der Auflösung 125 x 125 herangezogen und analysiert. Für alle Gefahrenkarten werden die Ergebnisse der GIS-Analysen ebenso wie die Vulnerabilität in Nieder, Mittel und Hoch kategorisiert.

Die genaue Methodik zur Abschätzung der Gefährdung durch Naturgefahren wird in Möderl et al., 2008 dargestellt. Im Folgenden werden die wesentlichen Überlegungen zusammengefasst.

\subsection{1. Überflutung (h1)}

Als Grundlage für die Abschätzung der Gefährdung durch Überflutung wurden die Ergebnisse des Projektes „Hochwasser Risiko Zonierung Austria“ (HORA, Blöschl et al., 2006) verwendet. Ziel des Projektes HORA war die Ausweisung von Überschwemmungsgebieten für Hochwasserabflüsse (HQT) der Jährlichkeiten $\mathrm{T}=30, \mathrm{~T}=100$ und $\mathrm{T}=200$ Jahre bezogen auf das im Maßstab 1:500.000 erfasste österreichische Flussnetz mit einer Gesamtlänge von ca. $26.000 \mathrm{~km}$. Dies entspricht einer Bestimmung von Hochwasserabflüssen für mehr als 10.000 Gebiete. Das Projekt wurde vom Bundesministerium für Land- und Forstwirtschaft, Umwelt und Wasserwirtschaft und dem Versicherungsverband Österreich nach dem Katastrophenhochwasser 2002 ins Leben gerufen. Die hydrologischen Arbeiten zu Bestimmung der T-jährlichen Abflüsse wurden vom Institut für Wasserbau und Ingenieurhydrologie der TU Wien in Zusammenarbeit mit dem Ingenieurbüro Günter Humer in Geboltskirchen durchgeführt.

\subsubsection{Hangrutschung (h2)}

Um die Gefährdung durch Hangrutschungen abzuschätzen zu können, wurde - falls vorhanden - der Gefahrenzonenplan herangezogen. Anderenfalls wurde das Verfahren von Perotto-Baldiviezo et al., 2003 modifiziert angewandt, wobei Raster- daten, die Gefälle und Landnutzung repräsentieren, verwendet wurden.

\subsubsection{Lawinen (h3)}

In Österreich zählen Schneelawinen $\mathrm{zu}$ den wichtigsten $\mathrm{Na}$ turgefahren. Es gibt eine Reihe verschiedener Lawinentypen, die zwei häufigsten Arten sind jedoch Schneebretter und Lockerschneelawinen. Bei der Schneebrettlawine spielt der Aufbau der Schneedecke eine wichtige Rolle. Durch Neuschnee erhöht sich die Auflast der obersten Schneeschicht. Entlang einer Schichtgrenze kommt es schließlich zum Abgang des Schneebrettes aus Neuschnee auf dem Altschnee. Lockerschneelawinen entstehen auf kohäsionslosen (ungebundenen) Schneeschichten, entweder in Form von Trocken- oder Nassschneelawinen. $70 \%$ der Lawinen in Österreich sind Schneebretter (Höller, 2007).

Das Bundesland Tirol umfasst die meisten Lawineneinzugsgebiete (2.189 Einzugsgebiete, BMLFUW, 2007). Ein Lawinenabgang kann in drei Prozessbereiche eingeteilt werden: das Anbruchgebiet, die Sturzbahn (Bewegungsbahn der Lawine), und das Ablagerungsgebiet, wo die Lawine ausläuft und zum Stillstand kommt. Es gibt bereits zahlreiche Modelle zur Berechnung von Lawinengefahren (Christen et al., 2002 und Gruber, 2007). Die in diesem Artikel durchgeführte Analysemethode beruht einerseits auf dem Gefahrenzonenplan und andererseits auf einer qualitativen Abschätzung der theoretischen Anbruchgebiete der Lawine. Die qualitativen Abschätzungen der Gefährdung wird anhand der $\mathrm{Pa}$ rameter Hangneigung, Sonnenexposition und Bodenbedeckung berechnet und fließt ebenfalls in die Lawinengefahrenkarte ein.

\subsubsection{Mure (h4)}

Massenverlagerungen stellen in 
den Alpen relief- und gesteinsbedingt charakteristische Prozesse dar, sie prägen viele Täler und sind natürliche Prozesse. Neben Lawinen, Berg- und Felsstürzen zählen auch Muren zu Massenverlagerungen (Veit, 2002). Als Murgang wird ein schnell fließendes Gemisch von Wasser und Feststoffen bezeichnet, das in der Regel dem steilsten Gefälle folgend abläuft (Ahnert, 2003). Es gibt zahlreiche komplexe GIS-basierte Modelle zur Erstellung von Muren-Gefahrenzonenpläne, einen Überblick dazu findet man beispielsweise in (Yu et al., 2006). Für diese Analysen sind allerdings geologische Grundlagendaten sowie Daten zur Bodenbeschaffenheit erforderlich. Für das Einzugsgebiet, welches in dieser Studie untersucht wurde, sind keine detaillierte Boden- und Geologiedatensätze vorhanden. Deshalb wurde eine vereinfachte Methode - in Bereichen, wo kein Gefahrenzonenplan vorlag durchgeführt, die auf das Vorhandensein von Wildbächen beruht.

\subsection{Risikoabschätzung}

Nach der Definition von UN DHA, 1992 und Varnes, 1984 kann Risiko als Produkt von Gefahr $(\mathrm{H})$ und Vulnerabilität (V) berechnet werden. Für die Abschätzung des Risikos im gesamten Untersuchungsgebiet werden demzufolge die Vulnerabilitäts- und Gefahrenkarte zur Risikokarte verschnitten.

\section{Resultate und Diskussion}

\subsection{Karten am Beispiel Kitzbühel}

In Abbildung 4 ist exemplarisch für alle Gefährdungen die Risikokarte für Hangrutschungen im Gebiet der Gemeinde Kitzbühel dargestellt. Die Vulnerabilitätskarten wurden mit oben beschriebener Methodik erstellt. Daraus ergab sich, dass das Wasserversorgungs-
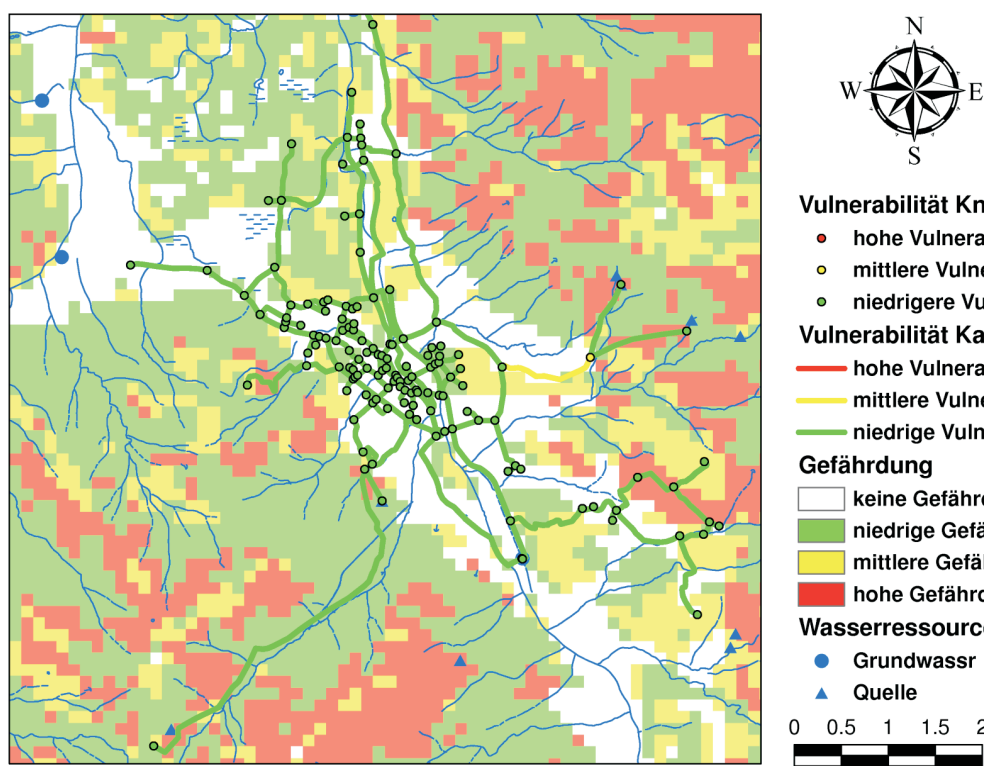

Vulnerabilität Knoten

- hohe Vulnerabilität

- mittlere Vulnerabilität

- niedrigere Vulnerabilität

Vulnerabilität Kanten

hohe Vulnerabilität

mittlere Vulnerabilität

- niedrige Vulnerabilität

Gefährdung

$\square$ keine Gefährdung

niedrige Gefährdung

mittlere Gefährdung

hohe Getährdung

Wasserressourcen

- Grundwassr

- Quelle

$\begin{array}{lllll}0 & 0.5 & 1 & 1.5 & 2\end{array}$

Abb. 4: Hangrutschungsgefahrenkarte und Vulnerabilitätskarte von Kitzbühel
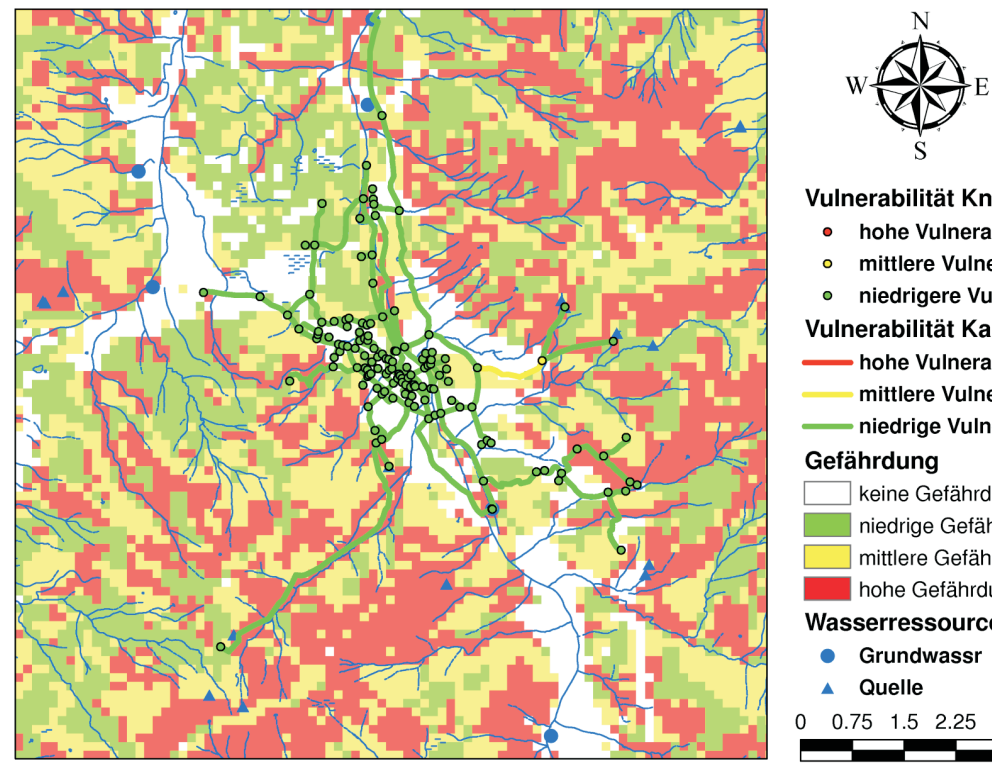

Vulnerabilität Knoten

- hohe Vulnerabilität

- mittlere Vulnerabilität

- niedrigere Vulnerabilität

Vulnerabilität Kanten

hohe Vulnerabilität

mittlere Vulnerabilität

niedrige Vulnerabilität

Gefährdung

$\square$ keine Gefährdung

niedrige Gefährdung

mittlere Gefährdung

hohe Gefährdung

Wasserressourcen

- Grundwassr

- Quelle

$\begin{array}{lllll}0 & 0.75 & 1.5 & 2.25 & 3\end{array}$

Abb. 5: Naturgefahrenrisikokarte von Kitzbühe

system der Gemeinde Kitzbühel im Vergleich mit den anderen Wasserversorgungssystemen generell wenig vulnerabel ist.

Im westlichen Teil des Versorgungsnetzes befinden sich $\mathrm{Ge}$ biete mit hohem Gefahrenpotential, jedoch sind die dort situierten Komponenten des Systems (auch Quellen) nicht von vorrangiger Wichtigkeit für das Systemverhalten d.h. dieser Teil ist wenig vulnerabel. Nur eine Zone des Netzes kann als risikoreich identifiziert werden, weil sich eine mittel vulnerable eingeschätzte Leitung in einem potentiell hohen Hangrutschungsgefahrenbereich befindet. Dieser Bereich sollte auf Basis von zusätzlich erhobenen Daten einer speziellen Analyse unterzogen werden. Falls diese detaillierte Analyse den gleichen Risikobefund als die hier präsentierten Ergebnisse zur folge hat, sollten Präventivmaßnahmen zur Minimierung des Ri- 
sikos gesetzt werden.

In Abbildung 2 ist die Naturgefahrenrisikokarte von Kitzbühel dargestellt, welche die Gefährdung von Überflutung, Hangrutschung, Lawinen und Muren inkludiert.

Gefährdung durch Überflutung besteht hauptsächlich an der Talsohle entlang der Kitzbüheler Ache. Ein Grundwasserbrunnen des Wasserversorgungssystems Kitzbühel liegt in einer hohen Überflutungsgefahrenzone, jedoch ist die Vulnerabilität dieses Brunnens gering. Die mittel vulnerable Leitung im Westen von Kitzbühel ist genauso wie durch Hangrutschungen auch durch Muren (h4) gefährdet.

\subsection{Gesamtheitliche qualitative Risikobewertung}

Insgesamt liegen 27 (das entspricht $0,42 \mathrm{~km}^{2}$ ) von 1.368 Rasterzellen in Zonen mit mittlerer und hoher Gefährdung durch Naturgefahren bzw. mittlerer und hoher Vulnerabilität. Es wird empfohlen diese 27 Zellen (d. h. die darin befindlichen Anlagen) einer speziellen Analyse zu unterziehen. Abhängig von dieser speziellen Tiefenanalyse können Präventivmaßnahmen gezielt eingesetzt werden.

\subsection{Detaillierte Betrachtung eines Netzbereiches mit hohem Risiko}

Exemplarisch wird in diesem Unterkapitel ein potentiell risikobehafteter Netzbereich im Detail betrachtet (Abbildung 6). Die Zone liegt in der Gemeinde Aurach und inkludiert drei hoch vulnerable und eine mittel vulnerabel eingestufte Wasserleitung in einer Murengefahrenzone (Einstufung hoch). Diese Leitungen sollen auf Basis profunder Datenlage und einer Vorortinspektion speziell analysiert werden, um die Unsicherheiten der qualitativen Analyse
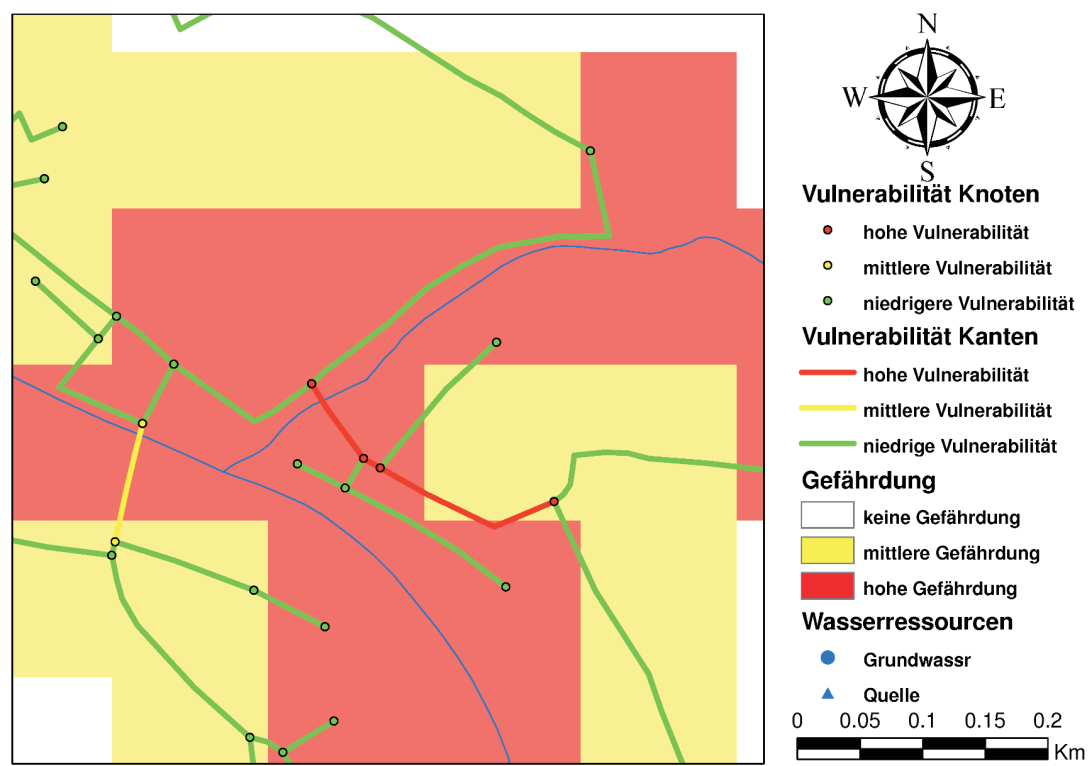

Abb. 6: Netzbereiche mit hohem Risiko (hervorgerufen durch Muren)

zu minimieren. Dabei kann eine Simulation der Murengefahr hilfreich sein. Wenn sich das aus der überregionalen Studie ergebende potentielle Risiko bestätigt kann z. B. durch Schutzeinrichtungen oder Erhöhung der Redundanz des Wasserversorgungssystems das Risiko verringert und dadurch die Versorgungssicherheit erhöht werden.

\section{Schlussfolgerungen}

In dieser Arbeit wurde eine neuartige Methodik zur qualitativen Abschätzung der Versorgungssicherheit von Wasserversorgungssystemen präsentiert. Die wesentliche Innovation ist dabei, dass nicht nur die Gefährdung des Systems (hier Naturgefahren) mit dem Leitungsnetz verschnitten wird, sondern die Systemvulnerabilität des Netzes detailliert und quantitativ betrachtet wird. Diese Systemvulnerabilität beziffert das Verhalten des Gesamtsystems bei Ausfall einer Komponente des Netzes (Leitung, Pumpe, Quelle, etc). Damit können punktgenau Zonen und Anlagen mit hohem Risiko bestimmt werden. Die Berechnung des Systemvulnerabi- lität erfolgt dabei mit dem am Arbeitsbereich Umwelttechnik, Universität Innsbruck entwickelten Softwarewerkzeug VulNetWS (Vulnerability of Water Supply Networks).

In einer Fallstudie wurden die Wasserversorgungen von $5 \mathrm{Ge}$ meinden in Tirol genau untersucht. Die Studie ergab, dass das Versorgungssystem der Gemeinde Kitzbühel wenig vulnerabel ist, verglichen mit den Systemen der anderen vier Gemeinden. Die erstellte Vulnerabilitätskarte repräsentiert dabei die Redundanz des Systems und nicht das Alter der Netzkomponenten. Zusätzlich wurde die Gefährdung durch Naturgefahren analysiert (Überflutungen, Hangrutschungen, Lawinen und Muren). Durch diese Gefahren sind im Untersuchungsgebiet insgesamt $0,42 \mathrm{~km}^{2}$ identifiziert worden die ein Risikopotential aufweisen und für eine lokale Detailstudie herangezogen werden sollen.

\section{Danksagung}

Diese Arbeit ist im Rahmen des Kompetenznetwerkes KNET (www.waterpool.org), Work Pa- 
ckage 2.1.2 „Alpine Wasserverund Vorsorgelogistik" entstanden. Es werden alle Projektpartnern und Fördergeber an dieser Stelle bedankt. Auch danken wir dem Bundesministerium für Landund Forstwirtschaft, Umwelt und Wasserwirtschaft (BMLFUW) für die Bereitstellung vom HORA-Datensatz.

\section{Korrespondenz:}

Michael Möderl, Arbeitsbereich Umwelttechnik, Universität Innsbruck, Technikerstraße 13, 6020 Innsbruck

Sara De Toffol, Arbeitsbereich Umwelttechnik, Universität Innsbruck, Technikerstraße 13, 6020 Innsbruck

Davy Vanham, Arbeitsbereich Umwelttechnik, Universität Innsbruck, Technikerstraße 13, 6020 Innsbruck Ernst Fleischhacker, Wasser Tirol - Wasserdienstleistungs-GmbH, Salurner Straße 6, 6020 Innsbruck

Wolfgang Rauch, Arbeitsbereich Umwelttechnik Universität Innsbruck, Technikerstraße 13, 6020 Innsbruck

\section{LITERATUR}

Ahnert, F (2003) Einführung in die Geomorphologie. Ulmer, Stuttgart, ISBN 978-3-8252-8103-8

BGBI 436/1976 (1976) Verordnung des Bundesministers für Land- und Forstwirtschaft vom 30. Juli 1976 über die Gefahrenzonenpläne

Blöschl G, Merz R, Humer G, Hofer M, Hochold A,

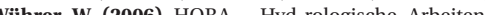
ogische Arbeiten. Wasserbau und ing

Christen M, Bartelt P, Gruber U (2002) AVAL-1D: An avalanche dynamics Program for the practice. In: Proceedings of the International Congress INTERPRAEVENT 2002 in the Pacific Rim - MATSUMOTO / JAPAN Congress publication, vol. 2, pp. 715-725

Ezell B C (2007) Infrastructure vulnerability assessment model (I-VAM). In: Risk Analysis, Vol. 27(3), p. 571-583 Gruber U (2007) Snow avalanche hazard modelling of large areas using shallow water nu-merical and GIS. In: Environmental Modelling and Software, Vol. 22(10, October 2007), p. 1472-1481

Höller P (2007) Avalanche hazards and mitigation in
Austria: a review. In: Natural Hazards, Vol. in press Kelman I (2003) Defining Risk. In: FloodRiskNet Newsletter,(2), p. 6-8

Möderl M, Fetz T, Rauch W (2007) Stochastic approach for performance evaluation re-garding wate perfistribuScience and Technology, Vol. p. $29-36$

Möderl M, Vanham D, De Toffol S, Rauch W (2008) Potential impact of natural haz-ards on water supply systems in Alpine regions. In: Proceedings of the World Water Congress and Exhibition, Vienna, 7-12.Sep, accepted

Perotto-Baldiviezo H L, Thurow T L, Smith C T, Fishe R F, Wu X B (2003) GIS-based spatial analysis and modeling for landslide hazard assessment in steeplands, southern Honduras. In: Agriculture, Ecosystems \& Environment, Vol. 103(1), p. 165-176

Rossman L A (2000) EPANET 2 user manual. Nation Risk Management Research Labo-ratory - U.S. ENVIRONMENTAL PROTECTION AGENCY

UN DHA (1992) Internationally Agreed Glossary of Basic Terms Related to Disaster Man-agement. UN DHA
(United Nations Department of Humanitarian Affairs) Vanham D, Fleischhacker E and Rauch W (2008) Technical Note: Seasonality in alpine water resources management - a regional assessment. In: Hydrology and Earth System Sci-ences, Vol. 12(1), p. 91-100

Vanham D, Millinger S, Heller A, Pliessig M, Möder M M, Rerfahren zu Erhebung und Anasse der Thinkwasserversorgungs-

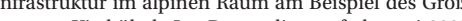
raumes Kitzbühel. In: Salz-burg, p. 832-837

Varnes D J (1984) Landslide hazard zonation: a review of principles and practice. Unesco, Paris, ISBN

Veit $\mathbf{H}$ (2002) Die Alpen - Geoökologie und Landschaftsentwicklung. Ulmer Verlag, Stutt-gart, ISBN 3-8001-2788-1

Yu F-C, Chen C-Y, Chen T-C, Hung F-Y, Lin S-C (2006) GIS Process for De-limitating Areas Potentially Endangered by Debris Flow. In: Natural Hazards, Vol. 37, p. $167-189$ 